\title{
On two cases of atypical respiratory distress in eastern Democratic Republic of the Congo months before the COVID-19 pandemic was declared. Could SARS-Cov-2 have been already spreading? Case report
}

\section{Guy-Quesney MATESO}

Department of Internal Medicine, Hôpital Provincial Général de Référence de Bukavu, Bukavu, DR Congo Marius BAGUMA ( $\sim$ baguma.akonkwa@ucbukavu.ac.cd)

Department of Internal Medicine, Hôpital Provincial Général de Référence de Bukavu, Bukavu, DR Congo ; Faculty of Medicine, Université Catholique de Bukavu (UCB), Bukavu, DR Congo https://orcid.org/00000002-4934-4846

\section{Pacifique MWENE-BATU}

Department of Internal Medicine, Hôpital Provincial Général de Référence de Bukavu, Bukavu, DR Congo; Faculty of Medicine, Université Catholique de Bukavu (UCB), Bukavu, DR Congo; École Régionale de Santé Publique (ERSP), Université Catholique de Bukavu (UCB), Bukavu, DR Congo. https://orcid.org/00000003-0413-2732

\section{Ghislain MAHESHE BALEMBA}

Department of Internal Medicine, Hôpital Provincial Général de Référence de Bukavu, Bukavu, DR Congo; Faculty of Medicine, Université Catholique de Bukavu (UCB), Bukavu, DR Congo; École Régionale de Santé Publique (ERSP), Université Catholique de Bukavu (UCB), Bukavu, DR Congo. https://orcid.org/00000003-2227-5999

\section{Fabrice NZABARA}

Department of Internal Medicine, Hôpital Provincial Général de Référence de Bukavu, Bukavu, DR Congo; Faculty of Medicine, Université Catholique de Bukavu (UCB), Bukavu, DR Congo

\section{Samuel MAKALI}

Department of Internal Medicine, Hôpital Provincial Général de Référence de Bukavu, Bukavu, DR Congo; Faculty of Medicine, Université Catholique de Bukavu (UCB), Bukavu, DR Congo; École Régionale de Santé Publique (ERSP), Université Catholique de Bukavu (UCB), Bukavu, DR Congo. https://orcid.org/00000003-3538-1715

\section{Aline BEDHA}

Department of Internal Medicine, Hôpital Provincial Général de Référence de Bukavu, Bukavu, DR Congo; Faculty of Medicine, Université Catholique de Bukavu (UCB), Bukavu, DR Congo

\section{Bonheur FURAHA}


Department of Internal Medicine, Hôpital Provincial Général de Référence de Bukavu, Bukavu, DR Congo Jimmy MINANI

Department of Internal Medicine, Hôpital Provincial Général de Référence de Bukavu, Bukavu, DR Congo https://orcid.org/0000-0001-8251-5582

\section{Christian TSHONGO MUHINDO}

Department of Internal Medicine, Hôpital Provincial Général de Référence de Bukavu, Bukavu, DR Congo; Faculty of Medicine, Université Catholique de Bukavu (UCB), Bukavu, DR Congo https://orcid.org/00000002-9511-7994

\section{Espoir BWENGE MALEMBAKA}

Department of Internal Medicine, Hôpital Provincial Général de Référence de Bukavu, Bukavu, DR Congo; Faculty of Medicine, Université Catholique de Bukavu (UCB), Bukavu, DR Congo; École Régionale de Santé Publique (ERSP), Université Catholique de Bukavu (UCB), Bukavu, DR Congo. https://orcid.org/00000001-9306-0253

\section{Mannix Imani MASIMANGO}

Department of Internal Medicine, Hôpital Provincial Général de Référence de Bukavu, Bukavu, DR Congo; Faculty of Medicine, Université Catholique de Bukavu (UCB), Bukavu, DR Congo

\section{Tony Akilimali SHINDANO}

Department of Internal Medicine, Hôpital Provincial Général de Référence de Bukavu, Bukavu, DR Congo; Faculty of Medicine, Université Catholique de Bukavu (UCB), Bukavu, DR Congo https://orcid.org/00000002-4473-8088

\section{Justin Cirhuza CIKOMOLA}

Department of Internal Medicine, Hôpital Provincial Général de Référence de Bukavu, Bukavu, DR Congo; Faculty of Medicine, Université Catholique de Bukavu (UCB), Bukavu, DR Congo https://orcid.org/00000002-0856-5992

\section{Kanigula MUBAGWA}

Faculty of Medicine, Université Catholique de Bukavu (UCB), Bukavu, DR Congo https://orcid.org/00000002-1028-4663

\section{Case Report}

Keywords: COVID-19, SARS-Cov-2 coronavirus, pandemic, DRCongo, Case report

Posted Date: May 20th, 2020

DOI: https://doi.org/10.21203/rs.3.rs-29541/v1

License: (a) (i) This work is licensed under a Creative Commons Attribution 4.0 International License. Read Full License 


\section{Abstract}

Background: Predictions have been made that Africa would be the most vulnerable continent to the novel coronavirus disease 2019 (COVID-19). Interestingly, the spread of the disease in Africa seems to be slower than in many parts of the World. Among possible hypotheses, some parts of Africa may have undergone a "silent" COVID-19 epidemic and acquired a herd immunity before the official declaration of the disease in December 2019.

Case presentation: We report two cases (one 55-year-old man and one 25-year-old woman) of acute respiratory distress secondary to atypical pneumonia in Bukavu, in eastern Democratic Republic of the Congo (DRC) which occurred between September and December 2019, before the official beginning of the COVID-19 pandemic. One patient had returned from China and the other had close contacts with travellers from China in the 2 weeks prior to the onset of symptoms. In either case, the aetiology could not be accurately determined. However, the two cases presented a clinical picture (progressive dyspnoea, preceded by dry cough and fever) and laboratory changes (procalcitonin within the normal range, slight inflammation, and lymphopenia) compatible with a viral infection. The chest X-Rays series of the first patient showed lesions (reticulations, ground glass, and nodules $\leq 6 \mathrm{~mm}$ ) similar to those found in COVID-19. In addition, unlike the 25-year-old female patient who had no comorbidity, the 55-year-old male patient who had hypertension as comorbidity, developed a more severe acute respiratory distress which progressed to death.

Conclusion: These cases bring to the attention a number of facts which make us suspect that the COVID19 epidemic may have already been present in the region months before the official beginning of the pandemic.

\section{Background}

The world is currently facing a devasting pandemic of the novel coronavirus disease 2019 (COVID-19), caused by severe acute respiratory syndrome coronavirus 2 (SARS-CoV-2), which was first declared in Wuhan, China, in December 2019 [1]. As of April 28, 2020, more than 3 million cases of COVID-19 have been confirmed in 185 countries, including more than 212,000 deaths [2]. Predictions have been made that Africa would be the most vulnerable continent to COVID-19, because of various factors: dense population in many cities, high prevalence of chronic diseases (e.g., infections by human immunodeficiency virus (HIV), malnutrition, etc.) interfering with the immune defence, absent or poor infrastructures to diagnose or treat patients, and very frequent travels between China and Africa $[3,4]$. The Democratic Republic of the Congo (DRC) is considered to be among the countries at highest risk, especially since the travels continued even after the pandemic was declared [3].

Interestingly, the spread of the disease in Africa seems to be slower than in many parts of the World. Since the first COVID-19 case was officially identified in Africa (in Egypt) on February 14, 2020, 22,239 confirmed cases and 881 deaths have been documented as of April 28, 2020, more than 2 months later 
[5]. These numbers are far below those recorded in other parts of the World. In the DRC, the first case has been reported on March 10, 2020, in Kinshasa (12 million inhabitants), but as of April 23, 2020, only 366 confirmed cases have been registered in the same city [6]. In Bukavu (about 1 million inhabitants, density: 16,600 inhabitants $/ \mathrm{km}^{2}$ ), the capital city of South-Kivu, in eastern DRC, the two first COVID-19 cases were travellers who arrived on March 18 and 20, but despite a delay in the isolation measures (started on March 30, 2020), more than a month later the Province has registered only 1 additional imported case.

Several reasons may explain this apparently low rate of COVID-19 propagation in Africa. Among possible hypotheses, some parts of Africa may have undergone a "silent" COVID-19 epidemic and acquired a herd immunity before the official declaration of the disease in December 2019. In this report, we present two patients who were admitted between September and December 2019 for acute respiratory distress in the Intensive Care Unit (ICU) of the "Hôpital Provincial Général de Référence de Bukavu" (HPGRB), in Bukavu. For both patients, a diagnosis of atypical viral pneumonia was made. Although they were not tested for COVID-19 (as the disease was not yet known at that time) the clinical presentation, radiological findings, and the course of the disease are compatible with COVID-19.

\section{Case Presentation}

Case 1: A 55-year-old man, with a 10-year history of hypertension and breeding pigeons for over five years, was seen in the Department of Internal Medicine on September 20, 2019 for dry cough, after returning from China 2 weeks before. A diagnosis of non-specific interstitial pneumonia was evoked based on a chest X-Ray (CXR) showing bi-basal and posterior densification (25-30\% of total chest height), blurring heart borders and diaphragm, associated with trabecular bands and ground-glass zones on the mid third of the lungs (Fig 1a, b). He was sent back home with oral azithromycin (500 mg daily for five days), acetaminophen and an antitussive drug.

Despite treatment, coughing was exacerbated, and fever and dyspnoea occurred two weeks later. On October 10, 2019 he was admitted in the Emergency ward. The clinical evaluation noticed polypnea (30 breaths $/ \mathrm{min})$, tachycardia $(100$ beats $/ \mathrm{min})$ and fever $\left(38^{\circ} \mathrm{C}\right)$. Oxygen saturation $\left(\mathrm{SaO}_{2}\right)$ was $54 \%$ [normal range: $95-100 \%$ ]. The patient presented a respiratory distress (nose flaring, chest retractions and cyanosis) and had fine crackles at both lung bases. Cardiovascular examination was normal. He had a moderate inflammatory syndrome with hyperleukocytosis $(11,600$ leucocytes $/ \mu \mathrm{l}[4,000-10,000 / \mu \mathrm{l}]$ of which 9,700 neutrophils $[1,500-7,000 / \mu \mathrm{l}]$ and 1,300 lymphocytes $[1,500-4,500 / \mu \mathrm{l}])$ and increased Creactive protein (CRP, $54.9 \mathrm{mg} / \mathrm{l}$ [0-3 mg/l]). Procalcitonin levels were normal [below $0.1 \mathrm{ng} / \mathrm{ml}$ ]. An arterial blood gas (ABG) revealed severe hypoxemia $\left(\mathrm{P}_{\mathrm{a}} \mathrm{O}_{2} 33 \mathrm{mmHg}[75-100 \mathrm{mmHg}]\right)$ and respiratory alkalosis (pH 7.58 [7.35-7.45], $\mathrm{P}_{\mathrm{a}} \mathrm{CO}_{2} 29.7 \mathrm{mmHg}$ [35-45 mmHg], $\mathrm{P}_{\mathrm{a}} \mathrm{O}_{2} / \mathrm{FiO}_{2}$ ratio $157 \mathrm{mmHg}$ [400-500 mm $\mathrm{mg}$ ], bicarbonate $27.1 \mathrm{mmol} / \mathrm{I}$ [22-26 mmol/l], and lactates $1.89 \mathrm{mmol} / \mathrm{l}[<2 \mathrm{mmol} / \mathrm{l}])$. Other laboratory results were normal. Bacteriological investigations were all negative. Tuberculosis was unlikely given a ZiehlNeelsen stain of sputum which was negative for three samples collected at different times. HIV serological tests were negative. A second CXR showed persistence of the aforementioned findings, except 
for the trabeculae hidden by the alveolar densification that had progressed up to the level of the main bronchi, overhung by a well delimited ground glass zone without encroachment upon the apices (Fig 1c).

The patient was hospitalized in the ICU for acute respiratory distress syndrome (ARDS) secondary to viral pneumonia with probable bacterial surinfection. He received oxygen by mask (5 litres/min) and intravenous antibiotics (combination of amoxycillin and clavulanic acid $1 / 0.25 \mathrm{~g}$ thrice daily). Despite this treatment, continuous fever (average temperature: $38.5^{\circ} \mathrm{C}$ ) and hypoxemia persisted, and lactates increased to $4 \mathrm{mmol} / \mathrm{l}$. So, two days later, amoxycillin and clavulanic acid were replaced by levofloxacin $(500 \mathrm{mg})$ and ceftriaxone $(1 \mathrm{~g})$, both twice daily, in addition to intravenous dexamethasone (16 mg thrice daily).

Four days later, no improvement was observed. The oxygen flow was increased to $10 \mathrm{litres} / \mathrm{min}$ and dexamethasone replaced by methylprednisolone ( $125 \mathrm{mg}$ twice daily for 5 days). During the following week, there was a slight improvement of the dyspnoea and fever, but on October 23, 2019, fever reappeared, and the respiratory distress worsened. A new CXR showed a reduction of the alveolar consolidation to the advantage of an interstitial syndrome (reticulations, ground-glass, and nodules $\leq 6$ $\mathrm{mm}$ ) extending to lung tops (Fig 1d). Meanwhile, the CRP was $49 \mathrm{mg} / \mathrm{l}$ and procalcitonin $0.62 \mathrm{ng} / \mathrm{ml}$. A diagnosis of respiratory zoonosis was considered, given the history of pigeon breeding. Intravenous methylprednisolone (125 mg twice daily) was reintroduced and Duovent ${ }^{\circledR}$ (combined ipratropium and fenoterol) was administered in nebulization, without any improvement. $\mathrm{SaO}_{2}$ remained at $40 \%$, $\mathrm{P}_{\mathrm{a}} \mathrm{O}_{2} / \mathrm{F}_{\mathrm{i}} \mathrm{O}_{2}$ ratio decreased to less than $70 \mathrm{mmHg}$, lactacidemia increased to $12.3 \mathrm{mmol} / \mathrm{l}$, and the patient became confused.

On October 25, 2019, the patient underwent orotracheal intubation for mechanical ventilation. This improved the $\mathrm{SaO}_{2}$ to $90-94 \%$ but on October 29, 2019, the patient developed a shock followed within a few hours by cardiac arrest irresponsive to resuscitation.

Case 2: A 25-year-old woman, with no history of recent travel, was admitted at the Emergency ward on December 18,2019 , for a one-week progressive dyspnoea, preceded by dry cough and fever. She had no particular medical history and was a nurse in a hospital where Chinese employees from a multinational mining company are treated, of whom some had recently travelled from China. On admission, she could not complete sentences due to dyspnoea. At ambient air, she presented a $\mathrm{SaO}_{2}$ of $82 \%$ and signs of respiratory distress without cyanosis. Her pulmonary auscultation was normal.

The CXR showed reticular lines and peribronchovascular haziness in the infrahilar and retrocardiac regions, bilaterally. This suggested a mild interstitial pneumonia (Fig 2a, b). Laboratory results showed a slight inflammation with CRP at $14.5 \mathrm{mg} / \mathrm{l}$, lymphopenia (700 lymphocytes/ $\mu \mathrm{l}$ ) and normal procalcitoninemia. The $A B G$ showed a hypoxemia $\left(\mathrm{P}_{\mathrm{a}} \mathrm{O}_{2} 60 \mathrm{mmHg}\right)$ and a respiratory alkalosis $(\mathrm{pH} 7.51$, $\mathrm{P}_{\mathrm{a}} \mathrm{CO}_{2} 35 \mathrm{mmHg}, \mathrm{P}_{\mathrm{a}} \mathrm{O}_{2} / \mathrm{F}_{\mathrm{i}} \mathrm{O}_{2} 286 \mathrm{mmHg}$, bicarbonate $27.1 \mathrm{mmol} / \mathrm{l}$, and lactates $\left.1.50 \mathrm{mmol} / \mathrm{l}\right)$. Creatinine, blood urea nitrogen and blood electrolytes were normal. HIV serology was negative. 
A diagnosis of moderate ARDS secondary to a viral pneumonia was retained and the patient was admitted in the ICU, receiving oxygen (4 litres/min), azithromycin ( $500 \mathrm{mg}$ once daily for 5 days) and Duovent ${ }^{\circledR}$ in nebulization. Three days later, she was eupnoeic with normal $\mathrm{SaO}_{2}$ at ambient air. She was discharged from hospital five days after admission.

\section{Discussion And Conclusion}

In this work, we report two cases of acute respiratory distress secondary to atypical pneumonia in eastern DRC. One patient had returned from China and the other had had close contacts with travellers from China in the 2 weeks prior to the onset of symptoms. In either case, the aetiology could not be accurately determined. COVID-19 was not yet known when these patients were hospitalized. However, when viewed retrospectively the two cases presented a clinical picture and laboratory changes (procalcitonin within the normal range, slight inflammation, and lymphopenia) compatible with a viral infection. The CXRs series of the first patient showed lesions (reticulations, ground glass, and nodules $\leq 6 \mathrm{~mm}$ ) similar to those found in COVID-19 [7]. In addition, unlike the 25-year-old female patient who had no comorbidity, the 55year-old male patient who had hypertension as comorbidity, developed a more severe acute respiratory distress which progressed to death. For COVID-19, available data show that older age and comorbidities such as cardiovascular diseases, diabetes, hypertension, chronic respiratory diseases and cancer are associated with an increased risk of death [8,9]. A diagnosis of psittacosis can hardly be sustained in view of the lack of response to antibiotics, and the dramatic evolution to death, uncommon in this disease. In retrospect, especially given the context of travel to China or of contact with travellers from China, the question can thus be raised as to whether these cases could have been COVID-19 infections.

The two cases and a number of other facts make us suspect that COVID-19 may have already been present in some regions of eastern DRC before the official beginning of the pandemic. The cases presented here are illustrative of (unpublished) observations made by clinicians in Bukavu during the last term of 2019. During that period, an abnormally high number of patients presented to local hospitals with symptoms of cough and fever, which were diagnosed as flu or atypical pneumonia for those who could have a chest radiography. Below are additional arguments that support the above hypothesis, although a confirmation cannot be provided.

First, the COVID-19 epidemic has apparently been late to hit Africa, relative to the starting dates in Asia or Europe. This is not due to lack of contacts with China, known to be the origin of the pandemic. During the past two decades business exchanges between many regions of Africa, especially the eastern part of the continent, with Asia, and in particular with China, have been on the increase [10]. Even after the official declaration of the pandemic, many persons from Africa continued to travel to China, to secure the import of various goods. The apparent delay in the outbreak of COVID-19 cannot therefore be explained solely on the grounds that there is a low level of traveling between Africa and China.

Secondly, since the local detection of a few positive cases, the progression of the number of such cases has been fortunately but astonishingly slow. The identification of positive cases only among travellers is 
usually taken to indicate that the epidemic is still at its initial (exogenous) stage before the phase of internal (indigenous community) transmission [11]. In more than a month, only 3 cases have been tested positive in South-Kivu. All of them were coming from travels outside of the country. Of a total of 32 known contacts of the positive cases, 18 have been tested and all turned out to be negative [6]. Surprisingly, contacts of one positive case include even very intimate partners, who on repeated testing have remained negative.

One reason for the small number of reported positive cases could be due to the low level of testing. However, indirect evidence of the local propagation of the disease could consist in the occurrence of an increased number of people consulting for cough, fever, dyspnoea and eventually for severe respiratory distress requiring respiratory assistance. Since the official declaration of the epidemic in the Province, in March 2020, such an increased incidence of acute respiratory diseases has not been observed locally, suggesting that either there is no rapid spread of COVID-19 or that there is indeed a spread but with less occurrence of severe cases, for example due protective factors such as the generally young age of the population, other immunizations (Bacille Calmette-Guérin vaccination, measles, etc.), some environmental or genetic factors, etc.

In view of the above-mentioned observations, it is tempting to suggest that SARS-CoV-2 infection might have already been present in the region. This hypothesis can only be examined by testing for the presence of anti-SARS-Cov-2 antibodies in the population. If this were to be true, then it would mean that most people have already been infected and eventually immunized, and that the most severe clinical cases have already died or been cured. This would also have important public health implications, as it would imply a loosening of preventive measures in the face of a putatively coming epidemic. Measures such as the compete confinement of the population (easily done in rich countries) are obviously difficult to implement in low-income countries because a large proportion of the population lives on daily income from selling or from seasonal jobs. If the infection were already here during the past year, this would raise the question as to whether the pandemic really started in China in December 2019.

New elements to disprove or support the hypothesis can come from the observed local evolution of the epidemic in the coming months. The occurrence of a severe epidemic would prove it wrong. However, only a retrospective analysis of blood samples from the population, initially targeting recalled known cases of atypical pneumonia but also common people would provide solid arguments.

This case report brings to the attention a number of facts suggesting that COVID-19 may have already been present in parts of eastern DRC (and possibly other parts of East Africa) months before the official start of the pandemic. Larger studies, including retrospective microbiological and serological investigations in local communities are needed to test this hypothesis.

\section{List Of Abbreviations}


ARDS : Acute respiratory distress syndrome

COVID-19 : The novel coronavirus disease 2019

CRP : C-reactive protein

CXR : Chest X-Rays

DRC : The Democratic Republic of the Congo

HIV : Human Immunodeficiency Virus

HPGRB : Hôpital Provincial Général de Référence de Bukavu

ICU : Intensive Care Unit

$\mathrm{SaO}_{2} \quad$ : Oxygen saturation

SARS-CoV-2 : Severe acute respiratory syndrome coronavirus 2

\section{Declarations}

\section{Ethics approval and consent to participate}

All patients admitted in the "Hôpital Provincial Général de Référence de Bukavu" consent that their clinical and imaging data can be used for publication or education purpose. All procedures performed were in accordance with the ethical standards of the institutional ethical committee and with the 1964 Helsinki declaration and its later amendments

All patients admitted consent that their clinical and imaging data can be used for publication or education purpose.

\section{Consent for publication}

Written informed consent for publication of the clinical details and/or clinical images was obtained from the patients or the next of kin (for the deceased patient).

\section{Availability of data and materials}

All data generated or analysed during this study are included in this manuscript.

\section{Competing interests}

The authors declare that they have no competing interests

\section{Funding}


There was no funding source for this study.

\section{Authors' contributions}

All authors contributed to the study conception and design. GQM, PM, SM, AB and BF contributed to the clinical patient care and management. Chest X-Rays were performed and interpreted by FN and GM. All authors contributed to the literature search. The first draft of the manuscript was written by MB and PM and all authors contributed to subsequent versions of the manuscript. All authors read and approved the final manuscript.

\section{Acknowledgements}

Not applicable.

\section{References}

1. Zhu N, Zhang D, Wang W, Li X, Yang B, Song J, et al. A Novel Coronavirus from Patients with Pneumonia in China, 2019. N Engl J Med. 2020;382:727-33. doi:10.1056/NEJMoa2001017.

2. Johns Hopkins University. COVID-19 Dashboard by the Center for Systems Science and Engineering (CSSE) at Johns Hopkins University (JHU). Johns Hopkins Coronavirus Resource Center. 2020. https://coronavirus.jhu.edu/map.html. Accessed 28 Apr 2020.

3. Velavan TP, Meyer CG. The COVID-19 epidemic. Trop Med Int Heal. 2020;25:278-80.

4. Gilbert M, Pullano G, Pinotti F, Valdano E, Poletto $C$, Boëlle P, et al. Preparedness and vulnerability of African countries against importations of COVID-19: a modelling study. Lancet. 2020;395:871-7.

5. World Health Organization (WHO). Coronavirus disease 2019 (COVID-19) Situation Report - 97. 2020; April. https://www.who.int/emergencies/diseases/novel-coronavirus-2019/situation-reports. Accessed 29 Apr 2020.

6. Ministère de la Santé Province du Sud-Kivu. Bulletin épidémiologique COVID-19 Sud-Kivu N 15/2020 du 23 Avril 2020. 2020. https://www.sudkivu.cd/gouv/wpcontent/uploads/2020/04/bulletin_epid_covid-19_sud-kivu-n¹5_2020-1.pdf. Accessed 29 Apr 2020.

7. Jacobi A, Chung M, Bernheim A, Eber C. Portable chest X-ray in coronavirus disease-19 ( COVID-19): A pictorial review. Clin Imaging. 2020;64 April:35-42. doi:10.1016/j.clinimag.2020.04.001.

8. Chen T, Wu D, Chen H, Yan W, Yang D, Chen G, et al. Clinical characteristics of 113 deceased patients with coronavirus disease 2019: retrospective study. BMG. 2019;368.

9. Guan W, Liang W, Zhao Y, Liang H, Chen Z, Li Y, et al. Comorbidity and its impact on 1590 patients with Covid-19 in China: A Nationwide Analysis. 2020.

10. Tull DM. China's engagement in Africa: Scope, significance and consequences. J Mod Afr Stud. 2006;44:459-79.

11. Indian council of medical research Department of Health Research - Ministry of Health \& Family Welfare Government of India. Media report on "Briefing on COVID19." 2020. 
https://www.icmr.nic.in/sites/default/files/MediaReport_COVID19.pdf. Accessed 27 Apr 2020.

\section{Figures}
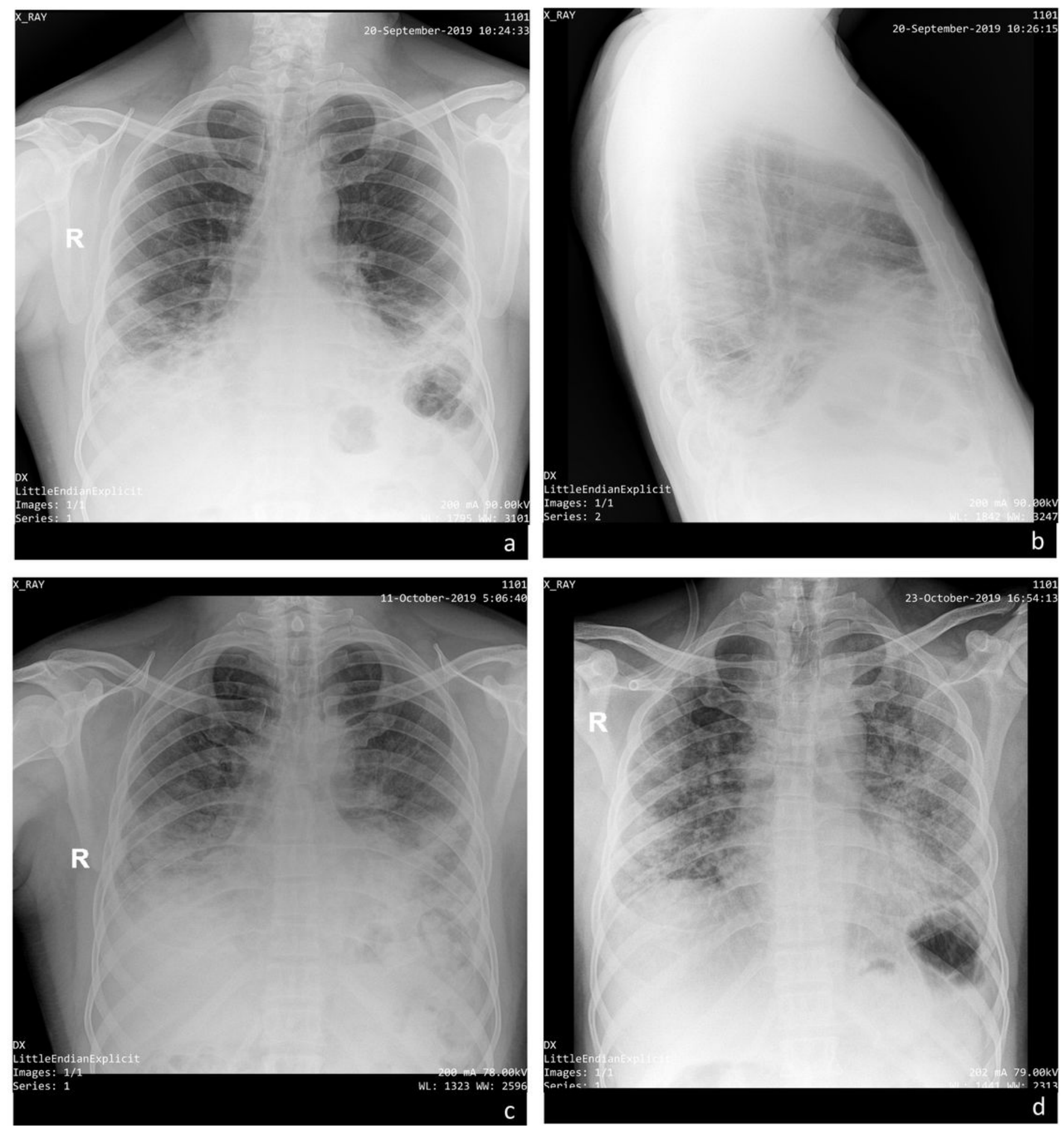

Figure 1 
Serial Chest X-Rays of Patient 1. Multifocal then diffuse airspace disease in a 55-years-old man. Bibasal lung consolidation with assorted trabeculae, especially in the posterior regions, overhung by discreet hazy opacities $(a, b)$. Follow-up showing a cranially progress of the predominant inhomogeneous consolidation still coexisting with a clearly diffuse ground-glass (c). Notice the apparent replacement of alveolar densification by diffuse reticular interstitial marks with some few parahilar nodules (d)
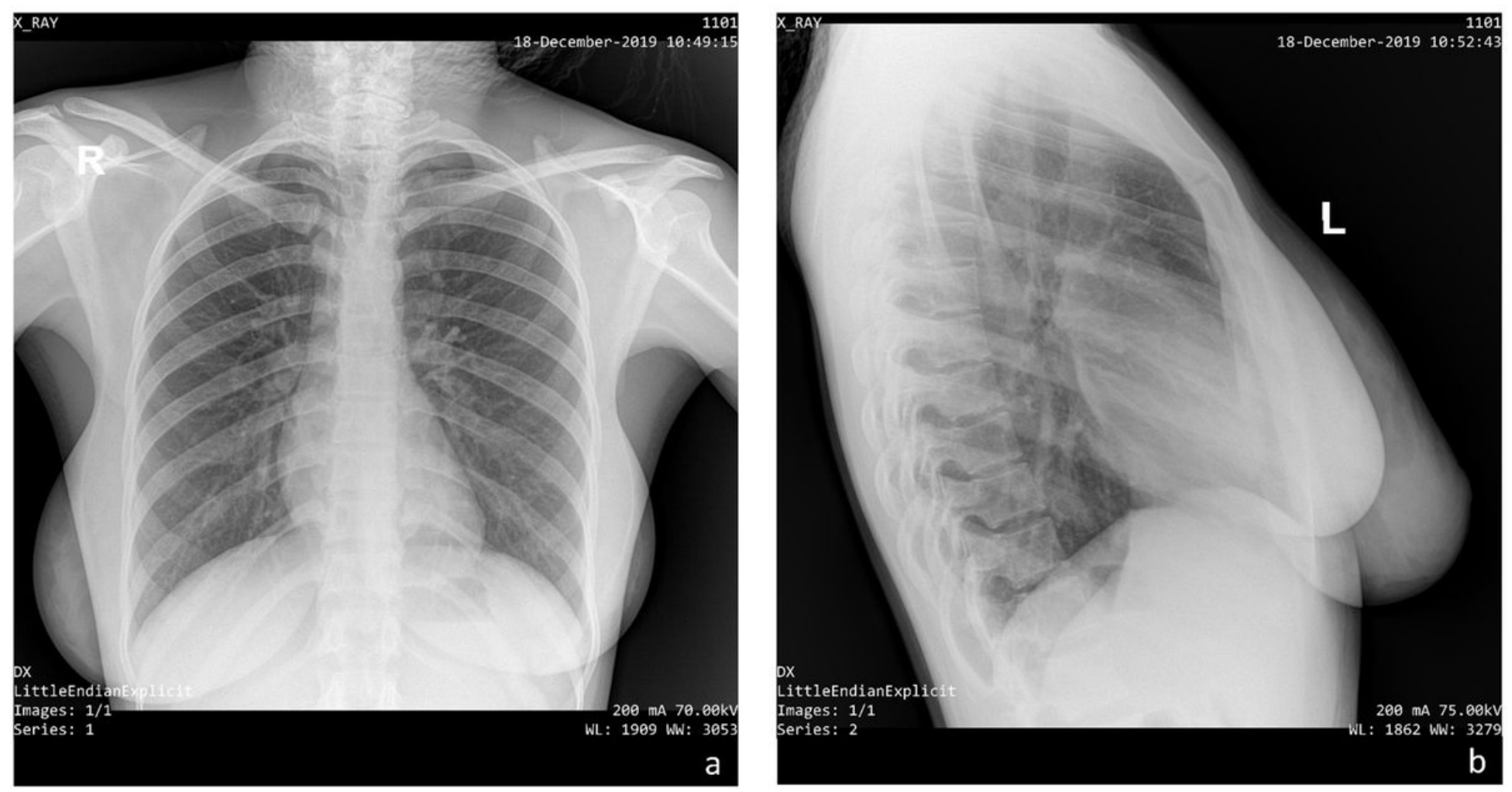

\section{Figure 2}

Chest X-Rays of Patient 2. Subnormal CXR of a 25-year-old female. Discrete peribronchovascular blur in the posterobasal regions. Normal aspect of the hila and the costophrenic angles. 


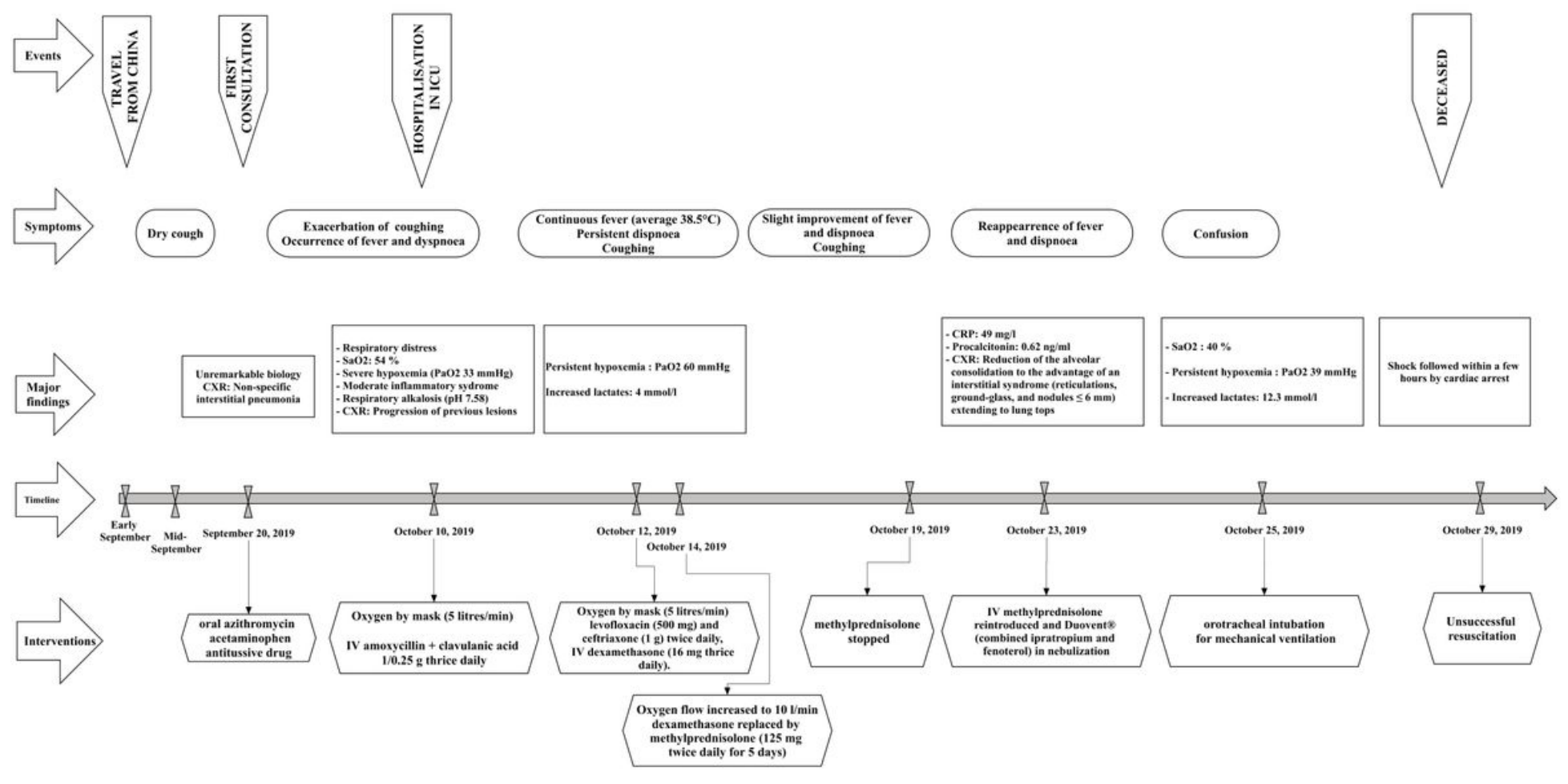

Figure 3

Summary of the 55-year-old patient's information and care organized as a timeline 

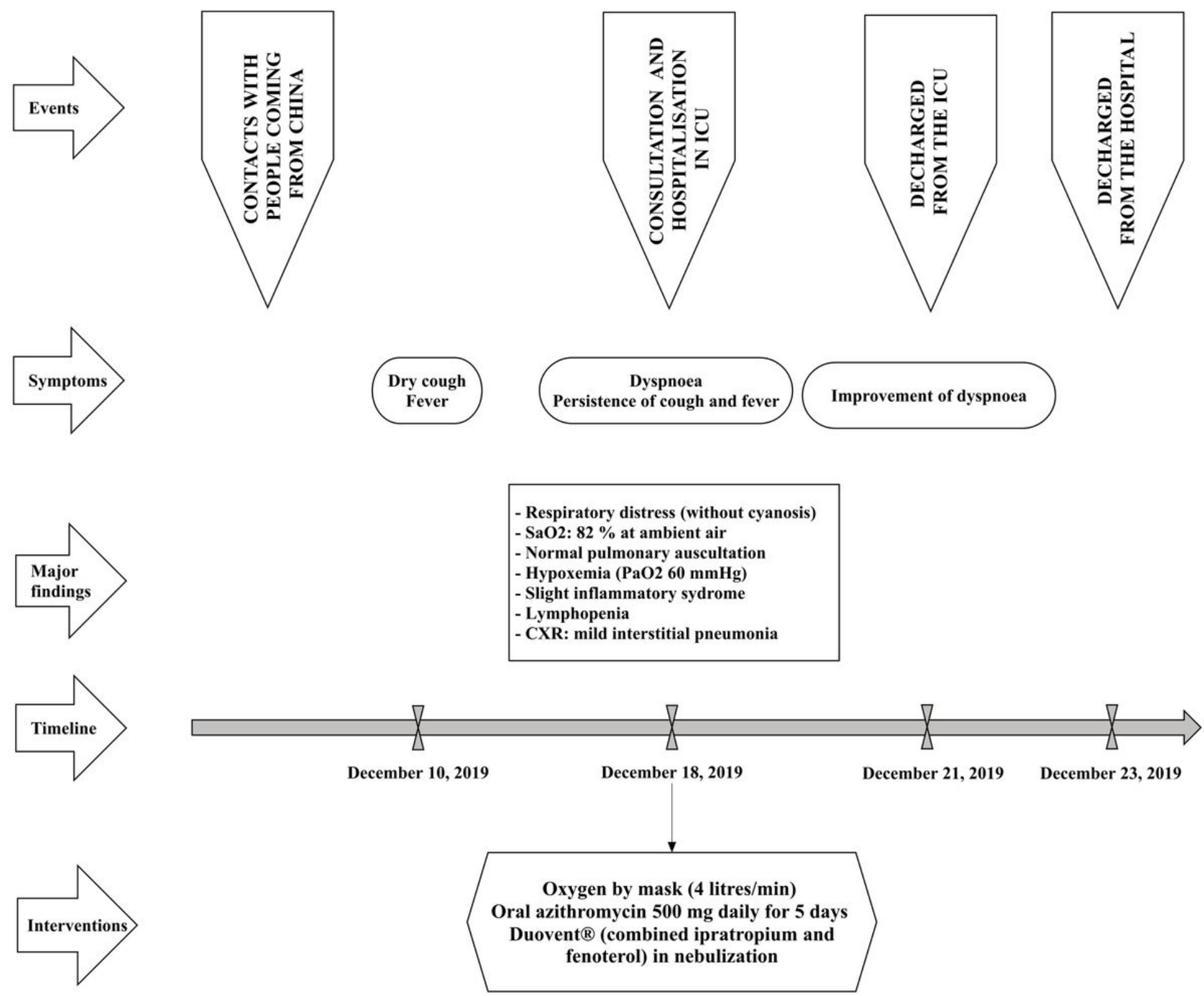

\section{Figure 4}

Summary of the 25-year-old patient's information and care organized as a timeline 